\title{
РЕГУЛЯРИЗОВАННЫЕ ПРИНЦИП ЛАГРАНЖА И ПРИНЦИП МАКСИМУМА ПОНТРЯГИНА В ОПТИМАЛЬНОМ УПРАВЛЕНИИ И ОБРАТНЫХ ЗАДАЧАХ ${ }^{1}$
}

\author{
М. И. Сумин
}

\begin{abstract}
Рассматривается регуляризация классических принципа Лагранжа и принципа максимума Понтрягина в выпуклом программировании, оптимальном управлении и обратных задачах. На примере "простейших" задач условной бесконечномерной выпуклой оптимизации обсуждаются два основных вопроса: зачем нужна регуляризация классических условий оптимальности (КУО) и что она дает? Так называемые регуляризованные КУО, о которых идет речь в статье, выражаются в терминах регулярных классических функций Лагранжа и Гамильтона-Понтрягина и являются секвенциальными обобщениями своих классических аналогов. Они: 1) "преодолевают" возможные неустойчивость и невыполнимость КУО, являясь регуляризирующими алгоритмами для решения оптимизационных задач; 2) формулируются как утверждения о существовании в исходной задаче ограниченных минимизирующих приближенных решений в смысле Дж. Варги и сохраняют общую структуру КУО; 3) приводят к КУО "в пределе". Все оптимизационные задачи в статье зависят от аддитивно входящего в бесконечномерное ограничение-равенство параметра (метод возмущений). Это позволило изучить связь регуляризованных КУО с субдифференциальными свойствами функций значений рассмотренных оптимизационных задач.
\end{abstract}

Ключевые слова: оптимальное управление, обратная задача, выпуклое программирование, метод возмущений, принцип Лагранжа, принцип максимума Понтрягина, двойственная регуляризация.

M. I. Sumin. Regularized Lagrange principle and Pontryagin maximum principle in optimal control and in inverse problems.

We consider a regularization of the classical Lagrange principle and Pontryagin maximum principle in convex programming, optimal control, and inverse problems. We discuss two basic questions, why a regularization of the classical optimality conditions (COCs) is necessary and what it gives, using the example of the "simplest" problems of constrained infinite-dimensional convex optimization. The so-called regularized COCs considered in the paper are expressed in terms of the regular classical Lagrange and Hamilton-Pontryagin functions and are sequential generalizations of their classical analogs. They (1) "overcome" the possible instability and infeasibility of the COCs, being regularizing algorithms for the solution of optimization problems, (2) are formulated as statements on the existence of bounded minimizing approximate solutions in the sense of Warga in the original problem and preserve the general structure of the COCs, and (3) lead to the COCs "in the limit." All optimization problems in the paper depend on an additive parameter in the infinite-dimensional equality constraint (the perturbation method). As a result, it is possible to study the connection of regularized COCs with the subdifferential properties of the value functions of the optimization problems.

Keywords: optimal control, inverse problem, convex programming, perturbation method, Lagrange principle, Pontryagin maximum principle, dual regularization.

MSC: 49K20, 49N15, 49N45, 47A52

DOI: $10.21538 / 0134-4889-2019-25-1-279-296$

\section{Введение}

Когда мы имеем дело с классическими условиями оптимальности (КУО), в частности, в задачах на условный экстремум, необходимо помнить о следующих двух важных обстоятельствах. Первое из них проявляется в характерном свойстве КУО, заключающемся в их неустойчивости и состоящем в том, что сколь угодно малым возмущениям исходных данных оптимизационной задачи могут отвечать сколь угодно большие возмущения выделяемых этими условиями элементов [1, введение; 2, введение]. Прежде всего, это является следствием неустойчивости самих оптимизационных задач, различные содержательные примеры которой

\footnotetext{
${ }^{1}$ Работа выполнена при поддержке РФФИ (проект 19-07-00782).
} 
можно найти, например, в [3, гл. 9]. В свою очередь, второе обстоятельство связано с возможной невыполнимостью КУО в разрешимых задачах условной оптимизации с бесконечномерными ограничениями (т. е. с ограничениями, задаваемыми операторами с бесконечномерными образами), различные примеры которой хорошо известны и могут быть найдены, в частности, в [1, введение; 2, введение; 4, п. 3.2.4, с. 260]. Оба этих обстоятельства в полной мере характерны для задач оптимального управления. Задачи оптимального управления, для которых характерны неустойчивость, невыполнимость классических принципа Лагранжа (ПЛ) и принципа максимума Понтрягина (ПМП), в большом числе возникают в различных важных естественнонаучных приложениях. $\mathrm{K}$ таким задачам следует, прежде всего, отнести задачи оптимального управления с фазовыми ограничениями. В частности, к задачам оптимального управления с фазовыми ограничениями-равенствами относятся разнообразные обратные задачи естествознания, без умения эффективно решать которые трудно представить современные научные исследования. Таким образом, оба указанных выше и связанных с КУО обстоятельства принципиально затрудняют использование классических ПЛ и ПМП в качестве непосредственных инструментов для решения многих сложных задач оптимального управления и сводящихся к ним разнообразных естественнонаучных обратных задач, в которых погрешности исходных данных жестко увязываются с физической сутью их постановок. Простые, но содержательные иллюстративные примеры задач оптимального управления и обратных задач, характеризующие как неустойчивость, так и невыполнимость КУО, см. в разд. 2.

Сказанное порождает мотивацию к такой естественной "корректировке" КУО, которая приводила бы к следующим двум “ожидаемым" свойствам: 1) “скорректированные" КУО должны "преодолевать" данные природой возможные неустойчивость и невыполнимость своих классических аналогов; 2) естественно желать, чтобы они были структурно устроены так же, как и сами КУО. Соображения самого общего характера позволяют заключить, что исправление данных природой "недостатков" классических ПЛ и ПМП в указанном направлении с необходимостью должно быть связано с применением методов теории регуляризации.

В статье показывается, как применение основанных на двойственности методов регуляризации [5-7] и одновременное использование в оптимизационной теории в качестве базового понятия минимизирующей последовательности допустимых элементов (в отличие от привычного понятия оптимального элемента) порождают такую естественную трансформацию КУО, которая приводит к их "ожидаемым" секвенциальным обобщениям. Эти выражаемые в терминах регулярных классических функций Лагранжа и Гамильтона - Понтрягина обобщения: 1) "преодолевают" возможные неустойчивость и невыполнимость КУО, являясь регуляризирующими алгоритмами для решения оптимизационных задач; 2) формулируются как утверждения о существовании в исходной (невозмущенной) задаче ограниченных минимизирующих приближенных решений в смысле Дж. Варги [8, п. III.2] и сохраняют общую структуру КУО; 3) приводят к КУО “в пределе". Такие трансформированные ПЛ и ПМП мы называем устойчивыми секвенциальными или, другими словами, регуляризованными ПЛ и ПМП $[1 ; 2 ; 7 ; 9]$. Тем самым трансформирование КУО в утверждения секвенциального характера, являющиеся одновременно устойчивыми к ошибкам исходных данных регуляризирующими алгоритмами решения задач, позволяет принципиально расширить сферу действия оптимизационной теории, основанной на привычных конструкциях функций Лагранжа и Гамильтона - Понтрягина.

Статья продолжает исследование вопросов регуляризации КУО в задачах оптимизации распределенных систем (см., например, [9]) и одновременно является расширенным вариантом работы [10], а также продолжением и расширением статьи [11]. В отличие от [9], здесь рассматриваются содержательные примеры неустойчивости и невыполнимости КУО, их теснейшая связь со своими классическими аналогами, а также регуляризованные КУО в итерационной форме. В свою очередь, в отличие от $[10 ; 11]$, в данной статье мы рассматриваем зависящие от аддитивно входящего в бесконечномерное ограничение-равенство параметра (метод возмущений $[4$, п. 3.3.2]) задачу выпуклого программирования (ВП) и сводимые к ней задачи оптимального управления, обратные задачи. Это позволило изучить здесь связь КУО, 
регуляризованных КУО с субдифференциальными свойствами функций значений задач, и показать, что КУО являются предельными вариантами своих регуляризованных аналогов при стремлении номеров элементов минимизирующих приближенных решений к бесконечности.

В целях более компактного изложения материала, как и в [10;11], мы рассматриваем "упрощенную" задача ВП и соответственно сводимые к ней задачу оптимального управления и обратную задачу. Упрощенность задачи ВП характеризуется, во-первых, заданием конкретного простейшего функционала качества и, во-вторых, отсутствием ограничений-неравенств. С одной стороны, благодаря упрощенной постановке рассматриваемая задача удобна для формулировки регуляризованных КУО, которые в этом случае получаются также более компактными по записи и, как следствие, более удобными для понимания. С другой же стороны, они достаточно полно передают основной содержательный смысл аналогичных результатов для более общих по форме оптимизационных задач на условный экстремум.

\section{1. “Простейшая" параметрическая задача ВП}

Рассмотрим "простейшую" параметрическую задачу (т. е. семейство задач, зависящих от параметра) ВП

$\left(P_{p}^{\delta}\right)$

$$
\|z\|^{2} \rightarrow \min , \quad A^{\delta} z=h^{\delta}+p, \quad z \in \mathcal{D} \subset Z .
$$

Здесь: $A^{\delta}: Z \rightarrow H-$ линейный ограниченный оператор, $h^{\delta} \in H-$ заданный элемент, $\mathcal{D} \subset Z$ - выпуклое замкнутое множество, $Z, H-$ гильбертовы пространства, $p \in H$ - параметр. Верхний индекс $\delta$ в исходных данных задачи $\left(P_{p}^{\delta}\right)$ означает, что эти данные являются точными $(\delta=0)$ или возмущенными $(\delta>0)$, т. е. задаются с ошибкой, величину которой и характеризует число $\delta \in\left[0, \delta_{0}\right]$, где $\delta_{0}>0-$ некоторое фиксированное число.

Предположим, что $\left\|\left(A^{\delta}-A^{0}\right) z\right\| \leq C \delta(1+\|z\|) \forall z \in Z,\left\|h^{\delta}-h^{0}\right\| \leq C \delta$, где $C>0$ не зависит от $\delta$. Обозначим единственное решение задачи $\left(P_{p}^{0}\right)$ в случае ее разрешимости через $z_{p}^{0}$.

3 а м е ч а н и е 1 . Подчеркнем, что если параметр $p \in H$ таков, что задача $\left(P_{p}^{0}\right)$ разрешима, то в силу линейности и непрерывности оператора $A^{0}$ и сильной выпуклости функционала качества $\|\cdot\|^{2}$ множество ее решений может быть только одноточечным. В свою очередь, в силу указанных причин факт разрешимости задачи $\left(P_{p}^{0}\right)$ равносилен факту разрешимости уравнения $A^{0} z=h^{0}+p$ на множестве $\mathcal{D}$.

Центральную роль ниже при рассмотрении задачи $\left(P_{p}^{0}\right)$ будет играть понятие минимизирующего приближенного решения в смысле Дж. Варги [8, п. III.2]. Определив, прежде всего, зависящую от параметра $p$ обобщенную нижнюю грань - (обобщенную) функцию значений $\beta(p)$ задачи $\left(P_{p}^{0}\right)$ как предел

$$
\beta(p) \equiv \lim _{\epsilon \rightarrow+0} \beta_{\epsilon}(p), \quad \beta_{\epsilon}(p) \equiv \inf _{z \in \mathcal{D}_{p}^{0, \epsilon}}\|z\|^{2}, \quad \beta_{\epsilon}(p)=+\infty, \quad \text { если } \mathcal{D}_{p}^{0, \epsilon}=\varnothing,
$$

где $\mathcal{D}_{p}^{\delta, \epsilon} \equiv\left\{z \in \mathcal{D}:\left\|A^{\delta} z-h^{\delta}-p\right\| \leq \epsilon\right\}, \epsilon \geq 0, \mathcal{D}_{p}^{0,0} \equiv \mathcal{D}_{p}^{0} \equiv\left\{z \in \mathcal{D}: A^{0} z-h^{0}-p=0\right\}$, напомним, что минимизирующим приближенным решением в задаче $\left(P_{p}^{0}\right)$ называется последовательность элементов $z^{k} \in \mathcal{D}, k=1,2, \ldots$, такая, что выполняются соотношения $\left\|z^{k}\right\|^{2} \rightarrow \beta(p)$, $z^{k} \in \mathcal{D}_{p}^{0, \epsilon^{k}}, k \rightarrow \infty$, для некоторой сходящейся к нулю последовательности $\epsilon^{k}, k=1,2, \ldots$, неотрицательных чисел. Из определения функции значений $\beta: H \rightarrow \mathbb{R}^{1} \cup\{+\infty\}$ следует, что, вообще говоря, $\beta(p) \leq \beta_{0}(p) \equiv\left\{\left\|z_{p}^{0}\right\|^{2}\right.$, если $z_{p}^{0}$ существует; $+\infty$ в противном случае $\} p \in H$, где $\beta_{0}: H \rightarrow \mathbb{R}^{1} \cup\{+\infty\}$ - классическая функция значений (классическая нижняя грань) задачи $\left(P_{p}^{0}\right)$. Однако специфика задачи $\left(P_{p}^{0}\right)$ такова, что справедлива

Лемма 1. Для функиии значений $\beta: H \rightarrow \mathbb{R}^{1} \cup\{+\infty\}$ имеет место равенство $\beta(p)=$ $\beta_{0}(p) \forall p \in H$, т.е. в задаче $\left(P_{p}^{0}\right)$ функиия значений $\beta$ совпадает с классической функиией значений $\beta_{0}$. Функция значений $\beta$ выпукла и полунепрерывна снизу. 
Д о к а з а т е л ь с т в о равенства обобщенной и классической функций значений проводится в точном соответствии со схемой доказательства полностью аналогичной леммы в [12, лемма 2.4.1]. Выпуклость $\beta$ доказана в [4, с. 264,265]), доказательство ее полунепрерывности снизу проводится полностью аналогично доказательству леммы 7 в [13, с. 34].

Лемма 2. Пусть $\beta(p)<+\infty$. Тогда для любого минимизирующего приблияснного решения $z^{k}, k=1,2, \ldots$, в разрешимой единственным образом в этом случае задаче $\left(P_{p}^{0}\right)$ справедливо предельное соотношение $z^{k} \rightarrow z_{p}^{0}, k \rightarrow \infty$.

Д о к а з а т е л ь с т в о. Во-первых, можно заметить, что так как $\beta(p)<+\infty$, а функционал $\|\cdot\|^{2}$ непрерывный и сильно выпуклый, то последовательность $z^{k}, k=1,2, \ldots$, о которой идет речь в теореме, является ограниченной. Во-вторых, считая ее без ограничения общности (в силу слабой компактности замкнутого шара в гильбертовом пространстве) слабо сходящейся и пользуясь слабой полунепрерывностью снизу функционалов $\|z\|^{2},\left\|A^{0} z-h^{0}-p\right\|, z \in \mathcal{D}$, получаем, что элементы $z^{k}$ при $k \rightarrow \infty$ сходятся слабо к решению $z_{p}^{0}$ задачи $\left(P_{p}^{0}\right)$. И, наконец, в-третьих, так как одновременно $\left\|z^{k}\right\|^{2} \rightarrow\left\|z_{p}^{0}\right\|^{2}$ при $k \rightarrow \infty$, то на основании классического $H$-свойства гильбертова пространства [14, п. 132, теорема 2] получаем сильную сходимость $z^{k}$ к $z_{p}^{0}$ при $k \rightarrow \infty$.

Определим функционал Лагранжа и связанные с ним необходимые ниже конструкции

$$
L_{p}^{\delta}(z, \lambda) \equiv\|z\|^{2}+\left\langle\lambda, A^{\delta} z-h^{\delta}-p\right\rangle, \quad z^{\delta}[\lambda] \equiv \operatorname{argmin}\left\{L_{p}^{\delta}(z, \lambda), z \in \mathcal{D}\right\}, \quad V_{p}^{\delta}(\lambda) \equiv \min _{z \in \mathcal{D}} L_{p}^{\delta}(z, \lambda),
$$

а также двойственную к $\left(P_{p}^{0}\right)$ задачу

$$
V_{p}^{0}(\lambda) \rightarrow \sup , \quad \lambda \in H, \quad V_{p}^{0}(\lambda) \equiv \min _{z \in \mathcal{D}} L_{p}^{0}(z, \lambda)
$$

\section{2. Классический ПЛ, его неустойчивость и невыполнимость}

Сформулируем, прежде всего, классический ПЛ в параметрической задаче $\left(P_{p}^{0}\right)$, напомнив предварительно, что вектором Куна - Таккера для нее называется элемент $\lambda \in H$, для которого имеет место неравенство $\beta(p) \leq L_{p}^{0}(z, \lambda) \forall z \in \mathcal{D}$.

Теорема 1 [Классический параметрический ПЛ]. Пусть $\beta(p)<+\infty$, m.e. задача $\left(P_{p}^{0}\right)$ разрешима (см. замечание 1 и лемму 2). Справедливы следующие утверждения.

1. Пусть $\zeta \in \partial \beta(p)$, где $\partial \beta(p)$ - субдифференииал в смысле выпуклого анализа. Тогда для оптимального элемента $z_{p}^{0} \in \mathcal{D}_{p}^{0} \equiv\left\{z \in \mathcal{D}: A^{0} z-h^{0}-p=0\right\}$ задачи $\left(P_{p}^{0}\right)$ и для множителя Лагранжа $\lambda \in H, \lambda=-\zeta$, при $\mu_{0}=1$ выполняется неравенство

$$
L_{p}^{0}\left(z_{p}^{0}, \mu_{0}, \lambda\right) \leq L_{p}^{0}\left(z, \mu_{0}, \lambda\right) \quad \forall z \in \mathcal{D}, \quad L_{p}^{0}\left(z, \mu_{0}, \lambda\right) \equiv \mu_{0}\|z\|^{2}+\left\langle\lambda, A^{0} z-h^{0}-p\right\rangle,
$$

и при этом $-\zeta=\lambda-$ вектор Куна - Таккера задачи $\left(P_{p}^{0}\right)$.

И наоборот, если $\tilde{z} \in \mathcal{D}_{p}^{0}-$ такой элемент, что при некоторых $\mu_{0}>0, \lambda \in H$ выполняется неравенство (2.1) с $\tilde{z}$ вместо $z_{p}^{0}$, то этот элемент оптимален в задаче $\left(P_{p}^{0}\right)$, m.е. $\tilde{z}=z_{p}^{0}$, элемент $\lambda / \mu_{0}$ является вектором Куна - Таккера для нее и одновременно $-\lambda / \mu_{0} \in \partial \beta(p)$.

2. Пусть $p \in \partial \operatorname{dom} \beta$ и $\zeta \in \partial^{\infty} \beta(p), \zeta \neq 0$, где $\partial^{\infty} \beta(p)-$ сингулярный (асимптотический) субдифференциал, определяемый формулой $\partial^{\infty} \beta(p) \equiv\left\{\lambda \in H:(\lambda, 0) \in N_{\text {epi }}(p, \beta(p))\right\}$ (см., например [15, definition 4C.1]). Тогда для оптимального элемента $z_{p}^{0} \in \mathcal{D}_{p}^{0}$ задачи $\left(P_{p}^{0}\right)$ и для множителя Лагранжа $\lambda \in H, \lambda=-\zeta$, неравенство (2.1) выполняется при $\mu_{0}=0$.

И наоборот, если $\tilde{z} \in \mathcal{D}_{p}^{0}-$ такой элемент, что при $\mu_{0}=0$ и некотором $\lambda \in H, \lambda \neq 0$, выполняется неравенство (2.1) с $\tilde{z}$ вместо $z_{p}^{0}$, то $p \in \partial \operatorname{dom} \beta$ и одновременно $-\lambda \in \partial^{\infty} \beta(p)$. 
Д о к а з а т е л ь с т в о более общего варианта теоремы см. в [1, теорема 2.1].

3 а м е ч а н и е 2. Представляет интерес сравнение на примере задачи $\left(P_{p}^{0}\right)$ в случае $\mathcal{D}=Z$ сформулированного в теореме 1 классического параметрического ПЛ с классическим ПЛ для гладких задач из книги $[4$, с. 253,254$]$. Последний применительно к задаче $\left(P_{p}^{0}\right)$ в

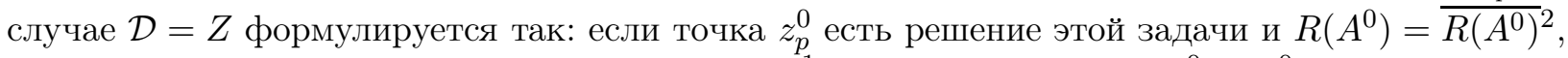
то найдется невырожденный набор $\left(\mu_{0}, \lambda\right) \in \mathbb{R}_{+}^{1} \times H$ такой, что $2 \mu_{0} z_{p}^{0}+A^{0 *} \lambda=0$, если же при этом $R\left(A^{0}\right)=H$, то в последнем равенстве можно считать $\mu_{0}>0$. Так как задача $\left(P_{p}^{0}\right)$ выпуклая, то в этом случае равенство $2 \mu_{0} z_{p}^{0}+A^{0 *} \lambda=0$ эквивалентно неравенству (2.1) при $\mathcal{D}=Z$. При этом можно заметить, что условие $R\left(A^{0}\right)=\overline{R\left(A^{0}\right)}, R\left(A^{0}\right) \neq H$ влечет наличие ненулевого элемента в сингулярном субдифференциале $\partial^{\infty} \beta(p)$, что в соответствии с первым утверждением второй части теоремы обеспечивает выполнимость нерегулярного $\left(\mu_{0}=0\right)$ ПЛ (2.1) при $\mathcal{D}=Z$. В то же время условие $R\left(A^{0}\right)=\overline{R\left(A^{0}\right)}=H$, как можно заметить, обеспечивает непустоту субдифференциала $\partial \beta(p)$ и, как следствие первого утверждения первой части теоремы 1 , выполнимость регулярного $\left(\mu_{0}>0\right)$ ПЛ $(2.1)$ при $\mathcal{D}=Z$. Таким образом, условия $R\left(A^{0}\right)=\overline{R\left(A^{0}\right)}, R\left(A^{0}\right) \neq H$ и $R\left(A^{0}\right)=\overline{R\left(A^{0}\right)}=H$ классического ПЛ для гладких задач $[4$, c. 253,254$]$ являются достаточными и для применимости соответствующих необходимых условий экстремума теоремы 1 . В то же время можно утверждать, что существует такой обширный класс задач, например вида $\left(P_{p}^{0}\right)$, для которого может быть записан невырожденный классический параметрический ПЛ теоремы 1 (т. е. в $(2.1)$ набор $\left(\mu_{0}, \lambda\right) \neq 0$ ), но, одновременно, не может быть применен ПЛ для гладких задач $[4$, с. 253,254$]$. В случае $\mathcal{D}=Z$ к таким задачам относятся, например, задачи, в которых $R\left(A^{0}\right) \neq \overline{R\left(A^{0}\right)}$, но, одновременно, либо $\partial^{\infty} \beta(p) \neq\{0\}$, либо $\partial \beta(p) \neq \varnothing$, либо и то и другое выполняется совместно. Прежде всего, к таким задачам можно отнести задачи с интегральными операторами $A^{0}$, для которых в большом числе важнейших с точки зрения различных естественнонаучных приложений случаев неравенство $R\left(A^{0}\right) \neq \overline{R\left(A^{0}\right)}$ выполняется. В частности, к ним относятся и задачи, рассмотренные ниже в примерах 1-3.

Второе утверждение первой части ПЛ теоремы 1 можно переписать в форме теоремы существования оптимального элемента с одновременным представлением последнего.

Теорема 2. [Теорема Куна - Таккера в форме теоремы существования оптимального элемента]. Если $z_{p}^{0} \in \mathcal{D}-$ такой элемент, что при некотором $\lambda \in H$ выполняются соотношения

$$
z_{p}^{0} \in \mathcal{D}_{p}^{0}, \quad L_{p}^{0}\left(z_{p}^{0}, \lambda\right) \leq L_{p}^{0}(z, \lambda) \quad \forall z \in \mathcal{D}, \quad L_{p}^{0}(z, \lambda) \equiv\|z\|^{2}+\left\langle\lambda, A^{0} z-h^{0}-p\right\rangle,
$$

то этот элемент оптимален в задаче $\left(P_{p}^{0}\right)$, элемент $\lambda$ является вектором Куна - Таккера для нее и одновременно $-\lambda \in \partial \beta(p)$.

3 а м е ч а н и е 3 . Последняя теорема сформулирована как достаточное условие существования оптимального элемента. Оно не требует существования вектора Куна - Таккера в задаче или, другими словами, не требует непустоты субдифференциала $\partial \beta(p)$. В то же время, как хорошо известно, нельзя утверждать, что если $z_{p}^{0} \in \mathcal{D}-$ оптимальный элемент в задаче $\left(P_{p}^{0}\right)$, то он с необходимостью удовлетворяет при некотором $\lambda$ неравенству в $(2.2)$. Таким образом, в этом случае выполнимость двух условий (2.2) при некотором $\lambda \in H$ является лишь достаточным для существования оптимального элемента, но, вообще говоря, не необходимым. Чтобы она стала и необходимым условием, надо потребовать существования вектора Куна Таккера в задаче $\left(P_{p}^{0}\right)$.

П р и м е р 1. Рассмотрим задачу $\left(P_{p}^{0}\right)$ с $H=Z, h^{0}=0$

$$
\|z\|^{2} \rightarrow \min , \quad A^{0} z=p, \quad z \in \mathcal{D} \subset Z,
$$

\footnotetext{
2 Здесь и ниже используется стандартное обозначение $R(A)$ для области значений оператора $A$.
} 
где $A^{0}: Z \rightarrow Z$ - линейный непрерывный инъективный оператор с инъективным сопряженным $A^{0 *}$. В этом случае, с учетом равенства $\left(A^{0 *}\right)^{*}=A^{0}$, имеют место равенства $\overline{R\left(A^{0}\right)}=Z$, $\overline{R\left(A^{0 *}\right)}=Z$ (см. [16, теорема 3.1]).

1.1. Неустойчивость ПЛ. Заметим, прежде всего, что если решение $z_{p}^{0}$ задачи $(P)$ удовлетворяет регулярному ПЛ в недифференциальной форме $L_{p}^{0}\left(z_{p}^{0}, \lambda\right) \leq L_{p}^{0}(z, \lambda) \forall z \in \mathcal{D}, L_{p}^{0}(z, \lambda) \equiv$ $\|z\|^{2}+\left\langle\lambda, A^{0} z-p\right\rangle, z \in \mathcal{D}$, теоремы 1 , то последнее неравенство может быть записано, за счет специфики "простейшей" задачи, в эквивалентной дифференциальной форме равенства $z_{p}^{0}=$ $\operatorname{Pr}_{\mathcal{D}}\left(-1 / 2 A^{0 *} \lambda\right)^{3}$. Данная эквивалентность двух форм ПЛ легко показывается, если записать условие минимума в точке $z_{p}^{0}$ в выпуклой гладкой задаче минимизации $L_{p}^{0}(z, \lambda) \rightarrow \min , z \in \mathcal{D}$, в привычной дифференциальной форме неравенства $\left\langle 2 z_{p}^{0}+A^{0 *} \lambda, z-z_{p}^{0}\right\rangle \geq 0 \forall z \in \mathcal{D}$, а затем заметить, что полученное неравенство означает в соответствии с классическим критерием проекции [3, гл. $4, \S 4$, теорема 1$]$, что точка $z_{p}^{0}$ есть проекция точки $-1 / 2 A^{0 *} \lambda$ на множество $\mathcal{D}$.

Заметим далее, что для любого элемента $\lambda \in H$ элемент $z^{0}[\lambda] \equiv \operatorname{argmin}\left\{L_{p}^{0}(z, \lambda), z \in \mathcal{D}\right\}$ удовлетворяет регулярному ПЛ в недифференциальной форме $L_{p}^{0}\left(z^{0}[\lambda], \lambda\right) \leq L_{p}^{0}(z, \lambda) \forall z \in \mathcal{D}$, в задаче $(P)$ с $p=A^{0} z^{0}[\lambda]$, эквивалентному в этом случае ПЛ в дифференциальной форме $z^{0}[\lambda]=\operatorname{Pr}_{\mathcal{D}}\left(-1 / 2 A^{0 *} \lambda\right)$, или (это в данной ситуации одно и то же) элемент $\lambda$ является вектором Куна - Таккера в рассматриваемой задаче и одновременно $-\lambda$ есть элемент субдифференциала выпуклой полунепрерывной снизу функции значений (как функции параметра $p$ ) задачи $(P)$, взятого в точке $p=A^{0} z^{0}[\lambda]$.

Пусть $p \in Z$ - любой такой элемент, для которого: 1) задача $(P)$ разрешима (очевидно, единственным образом); 2) это решение $z_{p}^{0}$ удовлетворяет при некотором $\lambda \in Z$ регулярному ПЛ в дифференциальной форме $z_{p}^{0}=\operatorname{Pr}_{\mathcal{D}}\left(-1 / 2 A^{0 *} \lambda\right) \equiv z^{0}[\lambda] \equiv \operatorname{argmin}\left\{L_{p}^{0}(z, \lambda), z \in \mathcal{D}\right\}$, и в эквивалентной недифференциальной форме $\left.L_{p}^{0}\left(z_{p}^{0}, \lambda\right) \leq L_{p}^{0}(z, \lambda) \forall z \in \mathcal{D} ; 3\right)$ существуют слабо, но не сильно сходящиеся в $Z$ к $z_{p}^{0}$ последовательности $z^{k} \in \mathcal{D}, k=1,2, \ldots$ (очевидно, такие последовательности заведомо существуют, если $\mathcal{D}=Z$ ), причем для всех таких последовательностей имеет место сильная сходимость $A^{0} z^{k} \rightarrow A^{0} z_{p}^{0}=p, k \rightarrow \infty$ (очевидно, последнее заведомо так, если оператор $A^{0}$ - вполне непрерывный).

Итак, пусть элементы $z^{k} \in \mathcal{D}, k=1,2, \ldots$, слабо, но не сильно сходятся при $k \rightarrow \infty \mathrm{k}$ $z_{p}^{0} \in \mathcal{D}$. Тогда $A^{0} z^{k} \rightarrow A^{0} z_{p}^{0} \equiv p, k \rightarrow \infty$ (сильно). Так как оператор $\operatorname{Pr}_{\mathcal{D}}$ действует на $\mathcal{D}$, то существует элемент $f^{k} \in Z$ такой, что $z^{k}=P r_{\mathcal{D}} f^{k}$. Естественно, в качестве элемента $f^{k}$ может быть взят сам элемент $z^{k}$. При этом так как $\overline{R\left(A^{0 *}\right)}=Z$, то для сколь угодно близких к $f^{k}$ элементов $\widetilde{f}^{k}$ выполняются равенства $\widetilde{f}^{k}=-1 / 2 A^{0 *} \widetilde{\lambda}^{k}$ при некотором $\widetilde{\lambda}^{k} \in Z$. Поэтому в силу непрерывности оператора проектирования для любого элемента $z^{k}$ найдется элемент $\lambda^{k} \in Z$, для которого соответствующий элемент $\widetilde{z}^{k} \equiv z^{0}\left[\lambda^{k}\right] \equiv \operatorname{Pr}_{\mathcal{D}}\left(-1 / 2 A^{0 *} \lambda^{k}\right)$ можно считать сколь угодно близким к $z^{k}$. Пусть $\epsilon^{k}, k=1,2, \ldots,-$ произвольная сходящаяся к нулю последовательность положительных чисел. Тогда, считая, что элемент $\lambda^{k}$ выбирается так, что $\left\|z^{k}-\widetilde{z}^{k}\right\| \leq \epsilon^{k}, k=1,2, \ldots$, получаем $\widetilde{z}^{k} \not \rightarrow z_{p}^{0}, k \rightarrow \infty$, но одновременно $A^{0} \widetilde{z}^{k} \equiv p^{k} \rightarrow p$, $k \rightarrow \infty$, и к тому же в соответствии со сказанным выше в каждой задаче $\|z\|^{2} \rightarrow \min , A^{0} z=$ $A^{0} \widetilde{z}^{k} \equiv p^{k}, \quad z \in \mathcal{D}$, решением которой является элемент $\widetilde{z}^{k}$, существует вектор Куна - Таккера или, другими словами, в точке $p^{k}$ не пуст субдифференциал функции значений задачи и, соответственно, выполняется регулярный ПЛ.

Следовательно, можно утверждать, что существуют такие $p^{k} \rightarrow p, k \rightarrow \infty$, для которых в аппроксимирующих (при $p=p^{k}$ ) задачах $(P)$ справедливо утверждение регулярного ПЛ такого же, как и в случае невозмущенной $(p=p)$ задачи $(P)$, но для которых одновременно оптимальные "аппроксимирующие" элементы не сходятся к решению невозмущенной задачи как по аргументу, так и по функции.

1.2. Невыполнимость ПЛ. Положим в задаче $(P): \mathcal{D} \equiv Z$. Предположим также, что оператор $A^{0}$ таков, что $R\left(A^{0 *}\right) \neq Z$ (последнее заведомо так, если оператор $A^{0}$ - вполне непре-

\footnotetext{
33десь и ниже запись $P r_{\mathcal{D}}$ означает обычный оператор проектирования на множество $\mathcal{D}$ в гильбертовом пространстве $Z$.
} 
рывный $\left[17\right.$, с. 225 , теорема 1]). Пусть при сделанных предположениях $\bar{z} \in Z \backslash R\left(A^{0 *}\right)-$ произвольный элемент. Тогда ПЛ в задаче $(P)$ при $p=A^{0}[\bar{z}]$ не выполняется. Предположим, что это не так. Тогда в соответствии с ПЛ теоремы 1 и с учетом показанной выше в этом примере равносильности двух форм ПЛ (см. также ПЛ для гладких задач $[4$, с. 253,254]) существует невырожденная пара множителей $\left(\mu_{0}, \lambda\right) \in \mathbb{R}_{+}^{1} \times Z$ такая, что $2 \mu_{0} \bar{z}+A^{0 *} \lambda=0$. В этом случае при $\mu_{0}=0$ получаем $\lambda=0$ в силу инъективности $A^{0 *}$, а при $\mu_{0}=1$ соответственно противоречивое равенство $\bar{z}=-1 / 2 A^{0 *} \lambda$ в силу неравенства $R\left(A^{0 *}\right) \neq Z$, что и доказывает невыполнимость ПЛ.

П р и м е р 2. Рассмотрим простейшую задачу оптимального управления с поточечным фазовым ограничением типа равенства, представляющую собой, по сути дела, простейшую обратную задачу

$$
\|u\|^{2} \rightarrow \min , \quad x[u]=p, \quad \dot{x}=u(t), \quad x(0)=0, \quad t \in(0,1), \quad u \in \mathcal{D},
$$

где $\mathcal{D} \in L_{2}(0,1)$ - выпуклое замкнутое множество, $p \in L_{2}(0,1)$ - параметр. Задача $(O C)$ является частным случаем задачи $(P)$ из примера 1 с $Z=L_{2}(0,1)$ и с линейными вполне непрерывными инъективными операторами:

$A^{0}, A^{0 *}: L_{2}(0,1) \rightarrow L_{2}(0,1), A^{0} u \equiv A^{0}[u], A^{0}[u](t) \equiv x^{0}[u](t) \equiv \int_{0}^{t} u(s) d s, A^{0 *}[u](t)=\int_{t}^{1} u(s) d s$.

Рассмотрим два варианта задачи $(O C)$.

2.1. Неустойчивость ПЛ и ПМП. Положим в задаче $(O C): p=0, \mathcal{D} \equiv\left\{u \in L_{2}(0,1): u(t) \in\right.$ $[-1,1]$ при п.в. $t \in(0,1)\}$. В этом случае решением задачи является $u_{0}(t) \equiv 0, t \in[0,1]$, причем, очевидно, существуют последовательности $u^{k} \in \mathcal{D}, k=1,2, \ldots$, такие, что $u^{k} \rightarrow u^{0}$, $k \rightarrow \infty$, слабо в $L_{2}(0,1)$, но не сильно, причем для всех таких последовательностей имеет место предельное соотношение $A^{0} u^{k} \rightarrow p=0, k \rightarrow \infty$. Так как выпуклая полунепрерывная снизу функция значений задачи $(O C)$ как функция $p$ субдифференцируема при $p=0$, то это оптимальное управление удовлетворяет при $p=0$ регулярному ПЛ

$$
L_{p}\left(u^{0}, \lambda\right) \leq L_{p}(u, \lambda) \forall u \in \mathcal{D}, \quad L_{p}(u, \lambda) \equiv\|u\|^{2}+\left\langle\lambda, A^{0} u-p\right\rangle, \quad u \in \mathcal{D},
$$

эквивалентному регулярному ПМП

$$
-\left(u^{0}(t)\right)^{2}-\eta(t) u^{0}(t)=\max _{v \in[-1,1]}\left\{-v^{2}-\eta(t) v\right\} \text { п.в. на }[0,1], \quad \dot{\eta}=\lambda(t), \quad \eta(1)=0 .
$$

Таким образом, проверена выполнимость всех условий 1)-3) примера 1.1. По этой причине можно утверждать, что существуют такие $p^{k} \rightarrow 0, k \rightarrow \infty$, для которых в аппроксимирующих задачах $(O C)$ при $p=p^{k}$ справедливы утверждения регулярных ПЛ и ПМП, аналогичных сформулированным выше для задачи $(O C)$ при $p=0$, но, одновременно, оптимальные "аппроксимирующие" управления не сходятся к решению невозмущенной задачи $(O C)$ как по аргументу, так и по функции.

2.2. Невыполнимость ПЛ. Положим далее $\mathcal{D} \equiv L_{2}(0,1)$. Очевидно, в этом случае $\overline{R\left(A^{0 *}\right)}=$ $L_{2}(0,1)$, но $R\left(A^{0 *}\right) \neq L_{2}(0,1)$. Пусть $\bar{u} \in L_{2}(0,1) \backslash R\left(A^{0 *}\right)$ - произвольный элемент. Тогда, как показано в примере 1.2 , ПЛ в задаче $(O C)$ при $p=x[\bar{u}]$ не выполняется.

П р и м е р 3. Рассмотрим далее классическую одномерную обратную задачу финального наблюдения по нахождению начальной функции $v \in \mathcal{D} \subset L_{2}(0,1), \mathcal{D}$ - выпуклое замкнутое множество, в третьей начально-краевой задаче для уравнения теплопроводности

$$
\begin{gathered}
z_{t}-z_{x x}=0, \quad z(x, 0)=v(x), \quad x \in \Omega \equiv(0,1), \\
z_{x}(0, t)-z(0, t)=0, \quad z_{x}(1, t)+z(1, t)=0, \quad t \in[0, T],
\end{gathered}
$$


которую можно трактовать также как задачу оптимального управления с фазовым ограничением типа равенства в финальный момент времени по нахождению начального управления в третьей начально-краевой задаче (2.3)

$$
\int_{0}^{1} v^{2}(x) d x \rightarrow \inf , \quad z[v](\cdot, T)=p \in L_{2}(0,1), \quad v \in \mathcal{D},
$$

где $z[v]$ - обобщенное решение [18, гл. III] начально-краевой задачи (2.3), соответствующее управлению $v \in \mathcal{D} \subset L_{2}(0,1)$. Задача $(I P)$ является частным случаем задачи $(P)$ из примера 1 с $Z=L_{2}(0,1)$ и с линейными непрерывными инъективными (инъективность может быть установлена, например, на основе результатов $[19, \S 2])$ операторами $A^{0}, A^{0 *}: L_{2}(0,1) \rightarrow L_{2}(0,1)$, $A^{0}[v](\cdot) \equiv z^{0}[v](\cdot, T), A^{0}[v] \equiv A^{0} v, A^{0 *}[\lambda](\cdot) \equiv \eta[\lambda](\cdot, 0), A^{0 *}[\lambda] \equiv A^{0 *} \lambda, \eta[\lambda]$ - соответствующее элементу $\lambda \in L_{2}(0,1)$ обобщенное решение сопряженной третьей краевой задачи

$\eta_{t}+\eta_{x x}=0, \quad \eta(x, 1)=\lambda(x), \quad x \in(0,1), \quad \eta_{x}(0, t)-\eta(0, t)=0, \quad \eta_{x}(1, t)+\eta(1, t)=0, \quad t \in[0, T]$.

3.1. Неустойчивость ПЛ и ПМП. Положим в задаче $(I P): p=0, \mathcal{D} \equiv\left\{v \in L_{2}(0,1)\right.$ : $v(x) \in[-1,1]$ при п.в. $x \in(0,1)\}$. В этом случае решением задачи является $v^{0}(x) \equiv 0, x \in$ $[0,1]$, причем, очевидно, существуют последовательности $v^{k} \in \mathcal{D}, k=1,2, \ldots$, такие, что $v^{k} \rightarrow v^{0}=0, k \rightarrow \infty$ слабо в $L_{2}(0,1)$, но не сильно; отметим, что для всех таких последовательностей имеет место предельное соотношение $A^{0} v^{k} \rightarrow p=0, k \rightarrow \infty$ (последнее имеет место в силу "равномерной гельдеровости" в этом случае обобщенных решений $z\left[v^{k}\right]$ в цилиндре $\{(x, t):[\epsilon, 1-\epsilon] \times[\epsilon, 1]\}$ при любом достаточно малом $\epsilon>0$, являющихся одновременно равномерно ограниченными на $[0,1] \times[0,1]$, см., например, [20, теорема 1$])$.

Так как выпуклая полунепрерывная снизу функция значений задачи $(I P)$ субдифференцируема при $p=0$, то указанное оптимальное управление удовлетворяет при $p=0$ и при некотором $\lambda \in L_{2}(0,1)$ регулярному ПЛ

$$
L_{p}\left(v^{0}, \lambda\right) \leq L_{p}(v, \lambda) \quad \forall v \in \mathcal{D}, \quad L_{p}(v, \lambda) \equiv\|v\|^{2}+\left\langle\lambda, A^{0} v-p\right\rangle, \quad v \in \mathcal{D},
$$

эквивалентному регулярному ПМП

$$
-\left(v^{0}(x)\right)^{2}-\eta[\lambda](x, 0) v^{0}(x)=\max _{v \in[-1,1]}\left\{-v^{2}-\eta[\lambda](x, 0) v\right\} \quad \text { при п.в. } x \in \Omega .
$$

Таким образом, проверена выполнимость всех условий 1)-3) примера 1.1 и, значит, относительно оптимального элемента $v^{0}$ задачи $(I P)$ можно сделать тот же самый вывод о его неустойчивости, что и для элемента $u^{0}$ в задаче $(O C)$ примера 2.1.

3.2. Невыполнимость ПЛ. Положим далее $\mathcal{D} \equiv L_{2}(0,1)$. Очевидно, в этом случае $\overline{R\left(A^{0 *}\right)}=$ $L_{2}(0,1)$, но $R\left(A^{0 *}\right) \neq L_{2}(0,1)$ (последнее неравенство имеет место в силу "заглаженности" решений краевых задач, см., например, [18, гл. III, теорема 8.1]). Пусть $\bar{v} \in L_{2}(0,1) \backslash R\left(A^{0 *}\right)-$ произвольный элемент. Тогда, как показано в примере 1.2 , ПЛ в задаче $(I P)$ при $p=z^{0}[\bar{v}]$ не выполняется.

\section{3. Регуляризованные ПЛ в “простейшей” задаче ВП}

Сформулируем и обсудим в данном разделе регуляризованные ПЛ $[1 ; 2 ; 21 ; 22]$ для задачи $\left(P_{p}^{0}\right)$. Их доказательства приведены в [11, теоремы $\left.3.1-3.3\right]$, а также в [21;22]. Они основаны на теоремах сходимости методов двойственной регуляризации и итеративной двойственной регуляризации с правилом останова итерационного процесса $[1 ; 2 ; 5 ; 6 ; 12 ; 21 ; 22]$. Соответствующие формулировки этих теорем сходимости также можно найти в [11, теоремы 2.1-2.3].

Формулируемые ниже регуляризованные ПЛ, которые можно также именовать регуляризованными теоремами Куна - Таккера (используемая функция Лагранжа регулярна) для 
задачи $\left(P_{p}^{0}\right)$, имеют вид утверждений о необходимых и достаточных условиях существования минимизирующего приближенного решения в задаче (подобно теореме 2 существования оптимального элемента) и о возможности аппроксимации решения $z_{p}^{0}$ точками минимума ее регулярной функции Лагранжа. Одновременно в них конструктивно предъявляются конкретные минимизирующие приближенные решения, аппроксимирующие решение $z_{p}^{0}$ (подобно тому как в теореме 2 предъявляется конкретный оптимальный элемент) и состоящие из указанных точек минимума регулярной функции Лагранжа.

Теорема 3 [Регуляризованный ПЛ]. Пусть задана произвольная последовательность сходящихся $к$ нулю положительных чисел $\delta^{k}, k=1,2, \ldots$ Для существования ограниченного минимизирующего приближенного решения в задаче $\left(P_{p}^{0}\right)$ необходимо и достаточно, чтобы существовала последовательность $\lambda^{k} \in H, k=1,2, \ldots$, такая, что выполняются соотношения

$$
\delta^{k}\left\|\lambda^{k}\right\|^{2} \rightarrow 0, \quad z^{\delta^{k}}\left[\lambda^{k}\right] \in \mathcal{D}_{p}^{\delta^{k}, \epsilon^{k}}, \quad \epsilon^{k} \rightarrow 0, \quad\left\langle\lambda^{k}, A^{\delta^{k}} z^{\delta^{k}}\left[\lambda^{k}\right]-h^{\delta^{k}}-p\right\rangle \rightarrow 0, \quad k \rightarrow \infty,
$$

а последовательность $z^{\delta^{k}}\left[\lambda^{k}\right], k=1,2, \ldots$, была ограничена. Более того, эта последовательность $z^{\delta^{k}}\left[\lambda^{k}\right], k=1,2, \ldots$, является искомым минимизирующим приближенным решением задачи $\left(P_{p}^{0}\right)$ и вне зависимости от того, разрешима или нет двойственная $\kappa\left(P_{p}^{0}\right)$ задача, $z^{\delta^{k}}\left[\lambda^{k}\right] \rightarrow z_{p}^{0}, k \rightarrow \infty$ (см. лемму 2). Кроме того, выполняется предельное соотношение $V_{p}^{0}\left(\lambda^{k}\right) \rightarrow \sup _{\lambda \in H} V_{p}^{0}(\lambda)=\left\|z_{p}^{0}\right\|^{2}, k \rightarrow \infty$. В случае существования ограниченного минимизирующего приближенного решения и разрешимости двойственной $\kappa\left(P_{p}^{0}\right)$ задачи $(\partial \beta(p) \neq \varnothing)$ можсно без ограничения общности считать, что $\lambda^{k} \rightarrow \lambda_{p}^{0}, k \rightarrow \infty$, где $\lambda_{p}^{0} \in H$ есть любое наперед выбранное и фиксированное решение указанной двойственной задачи (в частности, нормальное, т. е. минимальное по норме).

В качестве последовательности $\lambda^{k} \in H, k=1,2, \ldots$, может быть взята последовательность $\lambda_{p}^{\delta^{k}, \alpha\left(\delta^{k}\right)}, k=1,2, \ldots, \delta^{k} / \alpha\left(\delta^{k}\right) \rightarrow 0, k \rightarrow \infty$, генерируемая алгоритмом двойственной регуляризации, в соответствии с которым: $\lambda_{p}^{\delta, \alpha(\delta)} \equiv \operatorname{argmax}\left\{V_{p}^{\delta}(\lambda)-\alpha(\delta)\|\lambda-\widetilde{\lambda}\|^{2}\right.$, $\lambda \in H\}, \delta / \alpha(\delta) \rightarrow 0, \alpha(\delta) \rightarrow 0, \delta \rightarrow 0, \tilde{\lambda} \in H-$ произвольный фиксированный элемент. В случае разрешимости двойственной $\kappa\left(P_{p}^{0}\right)$ задачи $\lambda_{p}^{\delta, \alpha(\delta)} \rightarrow \lambda_{p}^{0}, \delta \rightarrow 0$, где в качестве $\lambda_{p}^{0} \in H$ может быть взято ее любое наперед выбранное и фиксированное решение (такая сходимость достигается за счет произвола в выборе $\widetilde{\lambda} \in H)$.

Д о к а з а т е л ь с т в о теоремы 3, которое проводится на основе метода двойственной регуляризации $[1 ; 2 ; 5 ; 6 ; 12 ; 21 ; 22]$, см. в [11, теорема 3.1$]$.

Сформулируем далее регуляризованный ПЛ в итерационной форме, который можно также именовать регуляризованной теоремой Куна - Таккера в итерационной форме. Приводимая формулировка, как и формулировка теоремы 3 , содержит необходимые и достаточные условия существования минимизирующего приближенного решения в задаче $\left(P_{p}^{0}\right)$. Однако в отличие от теоремы 3 в формулируемой ниже теореме, в основе доказательства которой лежит алгоритм итеративной двойственной регуляризации $[5 ; 6 ; 12 ; 21 ; 22]$, одновременное конструктивное предъявление конкретного минимизирующего приближенного решения, аппроксимирующего решение $z_{p}^{0}$ и состоящего из точек минимума регулярной функции Лагранжа, основано на итерационной процедуре регуляризованного градиентного подъема в процессе максимизации функционала $V_{p}^{0}$ двойственной задачи.

Введем в рассмотрение итерационный процесс

$$
\bar{\lambda}^{k+1}=\bar{\lambda}^{k}+\beta^{k}\left(A^{\delta^{k}} z^{\delta^{k}}\left[\bar{\lambda}^{k}\right]-h^{\delta^{k}}-p\right)-2 \beta^{k} \alpha^{k} \bar{\lambda}^{k}, \quad k=0,1,2, \ldots, \quad \bar{\lambda}^{0} \in H,
$$

с условиями согласования $\alpha^{k}>0, \beta^{k}>0, \lim _{k \rightarrow \infty}\left(\delta^{k}+\alpha^{k}+\beta^{k}\right)=0$,

$$
\frac{\alpha^{k}}{\alpha^{k+1}} \leq C_{0}, \quad \frac{\left|\alpha^{k+1}-\alpha^{k}\right|}{\left(\alpha^{k}\right)^{3} \beta^{k}} \rightarrow 0, \quad \frac{\beta^{k}}{\left(\alpha^{k}\right)^{3}} \rightarrow 0, \quad \frac{\delta^{k}}{\left(\alpha^{k}\right)^{6}} \rightarrow 0, \quad \sum_{k=1}^{\infty} \alpha^{k} \beta^{k}=+\infty .
$$


3 а м е ч а н и е 4. Последовательности $\alpha^{k}$ и $\beta^{k}, k=0,1,2, \ldots$, удовлетворяющие соотношениям (3.3), существуют. Например, в этом качестве можно использовать последовательности $\alpha^{k}=k^{-1 / 6}, \beta^{k}=k^{-1 /(5 / 3)}, k=0,1,2, \ldots$.

Теорема 4 [Регуляризованный итерационный ПЛ]. Для того чтобъ в задаче $\left(P_{p}^{0}\right)$ существовало ограниченное минимизирующее приближенное решение (u, следовательно, сильно сходилось $\left.к z_{p}^{0}\right)$, необходимо и достаточно, чтобы для последовательности $\bar{\lambda}^{k} \in H, k=$ $0,1, \ldots$, порождаемой итерационным прочессом (3.2) с условиями согласования (3.3), выполнялись соотношения

$$
z^{\delta^{k}}\left[\bar{\lambda}^{k}\right] \in \mathcal{D}_{p}^{\delta^{k}, \epsilon^{k}}, \quad \epsilon^{k} \rightarrow 0, \quad\left\langle\bar{\lambda}^{k}, A^{\delta^{k}} z^{\delta^{k}}\left[\bar{\lambda}^{k}\right]-h^{\delta^{k}}-p\right\rangle \rightarrow 0, \quad k \rightarrow \infty
$$

а последовательность $z^{\delta^{k}}\left[\bar{\lambda}^{k}\right], k=0,1, \ldots$, была ограниченной. В этом случае последовательность $z^{\delta^{k}}\left[\bar{\lambda}^{k}\right], k=0,1, \ldots$, представляет собой искомое минимизирующее приближсенное решение в задаче $\left(P_{p}^{0}\right)$ и вне зависимости от того, пуст субдифференциал $\partial \beta(p)$ или не пуст, имеет место сходимость $z^{\delta^{k}}\left[\bar{\lambda}^{k}\right] \rightarrow z_{p}^{0}, k \rightarrow \infty$ ( см. лемму 2). Одновременно выполняется $u$ предельное соотношение $V_{p}^{0}\left(\bar{\lambda}^{k}\right) \rightarrow \sup _{\lambda \in H} V_{p}^{0}(\lambda)=\left\|z_{p}^{0}\right\|^{2}, k \rightarrow \infty$. В случае существования ограниченного минимизирующего приблиюенного решения и разрешимости двойственной $\kappa\left(P_{p}^{0}\right)$ задачи $(\partial \beta(p) \neq \varnothing)$ можно без ограничения общности считать, что $\bar{\lambda}^{k} \rightarrow \lambda_{p}^{0}, k \rightarrow \infty$, где в качестве $\lambda_{p}^{0} \in H$ может быть взято ее любое наперед выбранное и фиксированное решение (в частности, нормальное).

Д о к а з а т е л ь с т в о теоремы 4, которое проводится на основе метода итеративной двойственной регуляризации $[5 ; 6 ; 12 ; 21 ; 22]$, см. в [11, теорема 3.2$]$.

3 а м е ч а н и е 5. Подчеркнем, что сформулированные регуляризованные теоремы Куна - Таккера 3, 4 отличаются от их классического аналога - классического параметрического ПЛ теоремы 1 - двумя важными обстоятельствами: 1) они справедливы без каких-либо предположений регулярности задачи $\left(P_{p}^{0}\right)$ (непустота субдифференциала, что равносильно существованию вектора Куна - Таккера, или наличие ненулевого элемента в сингулярном субдифференциале функции значений $\beta$ в точке $p$ ); 2 ) они “устойчивы" по отношению к ошибкам исходных данных в том смысле, что представляют собою регуляризирующие алгоритмы и тем самым могут использоваться, в частности, для решения некорректных задач, если соответствующая последовательность двойственной переменной выбирается согласно с алгоритмом двойственной регуляризации (теорема 3) или итеративной двойственной регуляризации (теорема 4). При этом важно то, что формулировки этих теорем обеспечивают одновременно как достаточное, так и необходимое условие существования минимизирующего приближенного решения в задаче. В этом состоит их принципиальное отличие от классического ПЛ (теоремы Куна - Таккера) в форме теорем 1 и 2.

Регуляризованный ПЛ в итерационной форме теоремы 4 может быть снабжен и регуляризирующим правилом останова итерационного процесса (3.2) в случае, когда исходные данные задачи $\left(P_{p}^{0}\right)$ задаются с фиксированной конечной ошибкой, характеризуемой величиной $\delta>0$. Это обстоятельство важно с точки зрения решения практических неустойчивых оптимизационных и сводящихся к ним задач. Тем самым оно обеспечивает возможность непосредственного применения регуляризованного итерационного ПЛ при решении самых разнообразных задач современного естествознания (см. [21;22], а также [23]). Пусть числовые последовательности $\delta^{k}, \alpha^{k}, \beta^{k}, k=0,1,2, \ldots$, удовлетворяют условиям (3.3). Зафиксируем следующее правило останова итерационного процесса (3.2):

$$
\bar{\lambda}^{k+1}=\bar{\lambda}^{k}+\beta^{k}\left(A^{\delta} z^{\delta}\left[\bar{\lambda}^{k}\right]-h^{\delta}-p\right)-2 \beta^{k} \alpha^{k} \bar{\lambda}^{k}, \quad k=0,1,2, \ldots ; \quad \bar{\lambda}^{0} \in H
$$


при фиксированном конечном уровне погрешности $\delta>0$ : при каждом $\delta>0, \delta \leq \delta^{1}$, итерации продолжаются до такого наибольшего номера $k=k(\delta)$, при котором выполняются неравенства

$$
\delta^{k} \geq \delta, \quad k=1,2, \ldots, k(\delta) .
$$

Тогда можно утверждать, что в дополнение к теореме 4 справедлива

Теорема 5. Вне зависимости от того, разрешима или нет двойственная $\kappa\left(P_{p}^{0}\right)$ задача, справедливы предельные соотношения $\left\|z^{\delta}\left[\bar{\lambda}^{k(\delta)}\right]-z_{p}^{0}\right\| \rightarrow 0, V_{p}^{0}\left(\bar{\lambda}^{k(\delta)}\right) \rightarrow \sup _{\lambda \in H} V_{p}^{0}(\lambda), \delta \rightarrow \infty$, где $\bar{\lambda}^{k(\delta)}$ - результат $k(\delta)$ итерачий итерационного процесса (3.5) с правилом останова, определяемым формулой (3.6). Таким образом, указанное правило останова порождает регуляризирующий алгоритм в задаче $\left(P_{p}^{0}\right)$.

Д о к а з а т е л ь с т в о теоремы 5 см. в [6, с. 622, теорема 3].

КУО являются предельными вариантами своих регуляризованных аналогов. КУО являются предельными вариантами регуляризованных КУО при стремлении номеров элементов минимизирующих приближенных решений к бесконечности. В этом смысле можно говорить, что регуляризованные КУО "содержат в себе" как частные случаи свои классические аналоги. Одновременно регуляризованные КУО вырождаются в пределе в случае невыполнимости своих классических аналогов. Для пояснения сказанного опять вернемся к "простейшей" задаче $\left(P_{p}^{0}\right)$. Действительно, в случае разрешимости двойственной задачи $(\partial \beta(p) \neq \varnothing)$ в соответствии с утверждениями теорем 3,4 , благодаря сильной сходимости соответствующих минимизирующих приближенных решений (напомним, что $z^{\delta}[\lambda] \equiv \operatorname{argmin}\left\{L_{p}^{\delta}(z, \lambda), z \in \mathcal{D}\right\}$ ) к оптимальному элементу $z_{p}^{0}$ и последовательности двойственной переменной к нормальному решению двойственной задачи $\lambda_{p}^{0}$, получаем в пределе при $k \rightarrow \infty L_{p}^{0}\left(z_{p}^{0}, \lambda_{p}^{0}\right) \leq L_{p}^{0}\left(z, \lambda_{p}^{0}\right) \forall z \in \mathcal{D}$ (из (3.1) в случае теоремы 3 и соответственно из (3.4) в случае теоремы 4). Если же $\partial \beta(p)=\varnothing$, но $\partial^{\infty} \beta(p) \neq\{0\}$, то для перехода к пределу в соотношениях теорем 3,4 поступаем несколько хитрее. Воспользуемся для этого двумя важными фактами, связанными со свойствами субдифференцируемости выпуклой полунепрерывной снизу функции значений $\beta$ (см. лемму 1 ). Первый из них заключается в том, что каждая такая функция в гильбертовом пространстве является субдифференцируемой на плотном множестве ее эффективного множества $[24$, теорема 4.3]. Второй же связан с известным представлением для асимптотического субдифференциала выпуклого полунепрерывного снизу функционала (см., например, [15, утверждение 4C2])

$$
\partial^{\infty} \beta(p)=\lim _{\substack{p^{\prime} \\ \rightarrow}, t \downarrow 0} \sup t \partial\left(p^{\prime}\right) \equiv\left\{w-\lim _{k \rightarrow \infty} t_{k} \zeta_{k}: t_{k} \downarrow 0, \zeta_{k} \in \partial \beta\left(p^{k}\right), p^{k} \stackrel{\beta}{\rightarrow} p\right\},
$$

где символ $p^{\prime} \stackrel{\beta}{\rightarrow} p$ означает, что $\left(p^{\prime}, \beta\left(p^{\prime}\right)\right) \rightarrow(p, \beta(p))$, а символ $t \downarrow 0$ означает сходимость к нулю справа. Тогда возьмем произвольную слабую предельную точку вида

$$
\tilde{\lambda}_{p}=w-\lim _{k \rightarrow \infty, p^{k} \stackrel{\beta}{\rightarrow} p, s_{k} \downarrow 0} s_{k} \lambda_{p^{k}}^{0}
$$

с $\lambda_{p^{k}}^{0} \in \partial \beta\left(p^{k}\right)$, причем в соответствии с теоремами 3 и 4 в данном случае в качестве $\lambda_{p^{k}}^{0}$ можно взять любой элемент из $\partial \beta\left(p^{k}\right)$. Так как в задаче с $p=p^{k}$ имеем $\partial \beta\left(p^{k}\right) \neq \varnothing$, то по доказанному выше можем записать $L_{p^{k}}^{0}\left(z_{p^{k}}^{0}, s_{k}, s_{k} \lambda_{p^{k}}^{0}\right) \leq L_{p^{k}}^{0}\left(z, s_{k}, s_{k} \lambda_{p^{k}}^{0}\right) \forall z \in \mathcal{D}$, где $L_{p}^{0}(z, s, \lambda) \equiv s\|z\|^{2}+$ $\left\langle\lambda, A^{0} z-h^{0}-p\right\rangle$. Переходя теперь в последнем неравенстве очевидным образом к пределу при $k \rightarrow \infty$ с учетом предельного соотношения $z_{p^{k}}^{0} \rightarrow z_{p}^{0}, k \rightarrow \infty$ (так как $\left\|z_{p^{k}}^{0}\right\|^{2} \rightarrow\left\|z_{p}^{0}\right\|^{2}$ в силу предельного соотношения $p^{k} \stackrel{\beta}{\rightarrow} p$ и $z_{p^{k}}^{0}$ слабо сходится к $z_{p}^{0}$ при $\left.k \rightarrow \infty\right)$, получаем неравенство $L_{p}^{0}\left(z_{p}^{0}, 0, \tilde{\lambda}_{p}\right) \leq L_{p}^{0}\left(z, 0, \tilde{\lambda}_{p}\right) \forall z \in \mathcal{D}$, означающее выполнимость нерегулярного ПЛ 
в задаче $\|z\|^{2} \rightarrow \min , A^{0} z=h^{0}+p, z \in \mathcal{D}$. Итак, регуляризованные ПЛ теорем 3,4 в пределе "приводят" к классическому ПЛ при любом $p \in \operatorname{dom} \beta$, для которого либо субдифференциал $\partial \beta(p)$ не пуст, либо, в случае его пустоты, асимптотический субдифференциал $\partial^{\infty} \beta(p)$ состоит не из одного нуля. Если же одновременно $\partial \beta(p)=\varnothing, \partial^{\infty} \beta(p)=\{0\}$, то регуляризованные принципы Лагранжа теорем 3, 4 в пределе вырождаются.

\section{4. Регуляризованные итерационные ПЛ и ПМП в оптимальном управлении и обратных задачах}

Основной целью данного раздела является иллюстрация того, как регуляризованные ПЛ разд. 3 могут применяться для решения неустойчивых задач оптимального управления и сводящихся к ним обратных задач. Формулируемая ниже задача оптимального управления с упрощенным функционалом качества может трактоваться одновременно как обратная задача финального наблюдения. Для нее формулируются регуляризованные ПЛ и ПМП в итерационной форме с правилом останова итерационного процесса [21-23;25;26].

Задача оптимального управления с фазовым ограничением-равенством. Пусть $Q_{T} \equiv \Omega \times(0, T), S \equiv \partial \Omega, S_{T} \equiv\{(x, t): x \in S, t \in(0, T)\}, \Omega-$ ограниченная область в $\mathbb{R}^{n}$, $H \equiv L_{2}(\Omega), \mathcal{D} \equiv \mathcal{D}_{1} \times \mathcal{D}_{2} \times \mathcal{D}_{3} \subset L_{2}\left(Q_{T}\right) \times L_{2}(\Omega) \times L_{2}\left(S_{T}\right) \equiv \mathcal{H}, \mathcal{D}_{1} \subset L_{2}\left(Q_{T}\right), \mathcal{D}_{2} \subset L_{2}(\Omega)$, $\mathcal{D}_{3} \subset L_{2}\left(S_{T}\right)$ - выпуклые замкнутые множества. Обозначим тройки элементов гильбертова пространства $\mathcal{H}$ через $\pi \equiv(u, v, w)$. Рассмотрим задачу оптимального управления с фиксированным временем и с операторным ограничением-равенством ${ }^{4}$

$\left(O C_{p}^{\delta}\right)\|\pi\|^{2} \equiv\|u\|_{2, Q_{T}}^{2}+\|v\|_{2, \Omega}^{2}+\|w\|_{2, S_{T}}^{2} \rightarrow \min , \quad A^{\delta} \pi \equiv z^{\delta}[\pi](\cdot, T)=h^{\delta}+p, \quad \pi \in \mathcal{D} \subset \mathcal{H} \equiv Z$.

Здесь: $h^{\delta} \in H$ - заданная функция, $p \in H-$ параметр, $z^{\delta}[\pi]$ - обобщенное решение класса $V_{2}^{1,0}\left(Q_{T}\right)[8]$ третьей начально-краевой задачи для линейного параболического уравнения с дивергентной главной частью ${ }^{5}$

$$
\begin{gathered}
z_{t}-\frac{\partial}{\partial x_{i}}\left(a_{i, j}^{\delta}(x, t) z_{x_{j}}\right)+a^{\delta}(x, t) z=u(x, t), \quad z(x, 0)=v(x), \quad x \in \Omega, \\
\frac{\partial z}{\partial \mathcal{N}}+\sigma^{\delta}(x, t) z=w(x, t), \quad(x, t) \in S_{T}
\end{gathered}
$$

соответствующее тройке $\pi \equiv(u, v, w) \in \mathcal{H}=Z, \partial z(x, t) / \partial \mathcal{N} \equiv a_{i, j}^{\delta}(x, t) z_{x_{j}}(x, t) \cos \alpha_{i}(x, t)$, $\alpha_{i}(x, t)$ - угол, образованный внешней нормалью к $S$ с осью $x_{i}$. Единственное решение задачи с точными исходными данными $\left(O C_{p}^{0}\right)$, если оно существует, будем обозначать через $\pi_{p}^{0}$. Заметим, что, как и в разд. 1 (см. замечание 1$)$, единственность решения $\pi_{p}^{0}$ является следствием разрешимости задачи, линейности и непрерывности оператора $A^{0}: \mathcal{H} \rightarrow H$ (см. следующий подраздел) и сильной выпуклости функционала $\|\pi\|^{2}, \pi \in \mathcal{D}$.

Считаем, что исходные данные задачи $\left(O C_{p}^{\delta}\right)$ удовлетворяют следующим условиям:

а) функции $a_{i, j}^{\delta}, a^{\delta}: \Omega \times[0, T] \rightarrow \mathbb{R}^{1}, i, j=1, \ldots, n, \sigma^{\delta}: S \times[0, T] \rightarrow \mathbb{R}^{1}$ являются измеримыми по Лебегу;

b) выполняются оценки $\nu|\xi|^{2} \leq a_{i, j}^{\delta}(x, t) \xi_{i} \xi_{j} \leq \mu|\xi|^{2} \quad \forall(x, t) \in Q_{T}, \quad \nu, \mu>0$,

$$
\left|a^{\delta}(x, t)\right| \leq K \text { при п.в. }(x, t) \in Q_{T},\left|\sigma^{\delta}(x, t)\right| \leq K \text { при п.в. }(x, t) \in S_{T},
$$

где $\nu, \mu, K>0$ - не зависящие от $\delta$ постоянные;

\footnotetext{
${ }^{4}$ Здесь и ниже символ $\|\varphi\|_{p, F}$ означает норму суммируемой со степенью $1 \leq p<\infty$ (измеримой существенно ограниченной в случае $p=\infty$ ) на множестве $F$ функции $\varphi$ в соответствующем функциональном пространстве.

5 Здесь и ниже при записи начально-краевых задач подразумевается, как и в [18, гл. III], суммирование от 1 до $n$ по парам повторяющихся индексов $i, j$.
} 
с) граница $S$ является кусочно-гладкой.

Будем считать также, что выполняются следующие оценки для отклонений возмущенных исходных данных от точных $(i, j=1, \ldots, n)$ :

$$
\left\|a_{i, j}^{\delta}-a_{i, j}^{0}\right\|_{\infty, Q_{T}} \leq \delta, \quad\left\|a^{\delta}-a^{0}\right\|_{\infty, Q_{T}} \leq \delta, \quad\left\|\sigma^{\delta}-\sigma^{0}\right\|_{\infty, S_{T}} \leq \delta, \quad\left\|h^{\delta}-h^{0}\right\|_{2, \Omega} \leq \delta .
$$

Регуляризованные итерационные ПЛ и ПМП. Задача $\left(O C_{p}^{\delta}\right)$ формально может быть записана как задача ВП, совпадающая по форме с задачей $\left(P_{p}^{\delta}\right)$ разд. $1: Z=\mathcal{H}, z \equiv \pi$, $H=L_{2}(\Omega), A^{\delta}: Z \rightarrow H-$ линейный ограниченный оператор, задаваемый равенством $A^{\delta} \pi=$ $z^{\delta}[\pi](\cdot, T), h^{\delta} \in L_{2}(\Omega), p \in L_{2}(\Omega)$. В силу условий а)-c) теорема существования обобщенного решения третьей начально-краевой задачи для линейного параболического уравнения с дивергентной главной частью $[20$, с. 34 , теоремы существования и единственности] (см. также $\left[18\right.$, гл. III, §5]) обеспечивает разрешимость прямой задачи $(4.1)$ в классе $V_{2}^{1,0}\left(Q_{T}\right)$ для любой тройки $(u, v, w) \in Z$ и любого $T>0$. Более того, мы имеем в этом случае и априорную оценку (напомним, что $|z|_{Q_{T}} \equiv \max _{0 \leq t \leq T}\|z(\cdot, t)\|_{2, \Omega}+\left\|z_{x}\right\|_{2, Q_{T}}$ )

$$
\left|z^{\delta}[\pi]\right|_{Q_{T}}+\left\|z^{\delta}[\pi]\right\|_{2, S_{T}} \leq C\left(\|u\|_{2, Q_{T}}+\|v\|_{2, \Omega}+\|w\|_{2, S_{T}}\right),
$$

в которой постоянная $C>0$ не зависит от $\delta \in\left[0, \delta_{0}\right]$ и $\pi \in \mathcal{H}=Z$. Из этой оценки следует, что линейный оператор $A^{\delta}: Z \rightarrow H=L_{2}(\Omega), A^{\delta} \pi=z^{\delta}[\pi](\cdot, T)$ при $\delta \in\left[0, \delta_{0}\right]$ является и непрерывным. Кроме того, используя ту же оценку в совокупности с оценками (4.2), получаем оценку для отклонения возмущенного $(\delta>0)$ оператора $A^{\delta}: Z \rightarrow H=L_{2}(\Omega), A^{\delta} \pi=z^{\delta}[\pi](\cdot, T)$ от его невозмущенного $(\delta=0)$ аналога в виде неравенства $\left\|\left(A^{\delta}-A^{0}\right) \pi\right\| \leq C \delta(1+\|\pi\|)$, в котором $C>0-$ постоянная, не зависящая от $\delta$ и $\pi \in \mathcal{H}=Z$. Определим множество $\mathcal{D}_{p}^{\delta, \epsilon} \equiv$ $\left\{\pi \in \mathcal{D}:\left\|A^{\delta} \pi-h^{\delta}-p\right\| \leq \epsilon\right\}$, функционал Лагранжа $L_{p}^{\delta}(\pi, \lambda) \equiv\|\pi\|^{2}+\left\langle\lambda, A^{\delta} \pi-h^{\delta}-p\right\rangle$, его единственный минимизирующий элемент $\pi^{\delta}[\lambda]$ (функционал качества задачи $\left(O C_{p}^{\delta}\right)$ является сильно выпуклым) и двойственную к $\left(O C_{p}^{\delta}\right)$ задачу $V_{p}^{\delta}(\lambda) \equiv \min _{\pi \in \mathcal{D}} L_{p}^{\delta}(\pi, \lambda) \rightarrow \sup , \lambda \in H=$ $L_{2}(\Omega)$.

Теорема 4 позволяет нам сформулировать ПЛ в итерационной форме для задачи оптимального управления $\left(O C_{p}^{\delta}\right)$. Его формулировка использует классическую конструкцию функционала Лагранжа и не зависит от того, существует или нет вектор Куна - Таккера в рассматриваемой задаче. Попутно заметим, что вопрос существования вектора Куна - Таккера в подобных задачах представляет собою самостоятельную сложную проблему.

Теорема 6 [Регуляризованный итерационный ПЛ]. Для существования ограниченного минимизирующего приближенного решения в задаче $\left(O C_{p}^{0}\right)$ необходимо и достаточно, чтобы для последовательности двойственной переменной $\bar{\lambda}^{k}, k=1,2, \ldots$, порождаемой итерационным прочессом

$$
\bar{\lambda}^{k+1}=\bar{\lambda}^{k}+\beta^{k}\left(A^{\delta^{k}} \pi^{\delta^{k}}\left[\bar{\lambda}^{k}\right]-h^{\delta^{k}}-p\right)-2 \beta^{k} \alpha^{k} \bar{\lambda}^{k}, \quad k=0,1,2, \ldots, \quad \bar{\lambda}^{0} \in H=L_{2}(\Omega),
$$

с условиями согласования (3.3), выполнялись соотношения

$$
\pi^{\delta^{k}}\left[\bar{\lambda}^{k}\right] \in \mathcal{D}_{p}^{\delta^{k}, \epsilon^{k}}, \quad \epsilon^{k} \rightarrow 0, \quad\left\langle\bar{\lambda}^{k}, A^{\delta^{k}} \pi^{\delta^{k}}\left[\bar{\lambda}^{k}\right]-h^{\delta^{k}}-p\right\rangle \rightarrow 0, \quad k \rightarrow \infty
$$

а последовательность $\pi^{\delta^{k}}\left[\bar{\lambda}^{k}\right], k=0,1, \ldots$, была ограниченной. Более того, последовательность $\pi^{\delta^{k}}\left[\bar{\lambda}^{k}\right], k=1,2, \ldots$, является искомым минимизирующим приближенным решением в задаче $\left(O C_{p}^{0}\right)$ и вне зависимости от того, разрешима или нет двойственная к $\left(\right.$ оC $\left.C_{p}^{0}\right)$ задача, $\pi^{\delta^{k}}\left[\bar{\lambda}^{k}\right] \rightarrow \pi_{p}^{0}, k \rightarrow \infty$ (см. лемму 2). Одновременно выполняется и предельное соотночение $V_{p}^{0}\left(\bar{\lambda}^{k}\right) \rightarrow \sup _{\lambda \in H} V_{p}^{0}(\lambda)=\left\|\pi_{p}^{0}\right\|^{2}, k \rightarrow \infty$. 
Естественно, мы можем сформулировать и соответствующее правило останова для итерационного процесса (4.3), представляющее собою регуляризирующий алгоритм в задаче $\left(O C_{p}^{0}\right)$, в случае, когда исходные данные задачи $\left(O C_{p}^{0}\right)$ задаются с фиксированной конечной ошибкой $\delta>0$. В этом случае следствием теоремы 5 является

Теорема 7. Имеют место пределъные соотношения $\left\|\pi^{\delta}\left[\bar{\lambda}^{k(\delta)}\right]-\pi_{p}^{0}\right\| \rightarrow 0, V_{p}^{0}\left(\bar{\lambda}^{k(\delta)}\right) \rightarrow$ $\sup V_{p}^{0}(\lambda)$ при $\delta \rightarrow \infty$, где $\bar{\lambda}^{k(\delta)}$ - результат $k(\delta)$ итераций итерационного прочесса (3.5) $\left(A^{\delta} z^{\delta}\left[\bar{\lambda}^{k}\right] \equiv A^{\delta} \pi^{\delta}\left[\bar{\lambda}^{k}\right]\right)$ с правилом останова, определяемым формулой (3.6).

Получим далее регуляризованный ПМП в итерационной форме из ПЛ в итерационной форме теоремы 6. Предположим для простоты, что множества $\mathcal{D}_{1}, \mathcal{D}_{2}, \mathcal{D}_{3}$ имеют более привычный для теории оптимального управления вид: $\mathcal{D}_{1} \equiv\left\{u \in L_{2}\left(Q_{T}\right): u(x, t) \in U\right.$ п.в. на $\left.Q_{T}\right\}$, $\mathcal{D}_{2} \equiv\left\{v \in L_{2}(\Omega): v(x) \in V\right.$ п.в. на $\left.\Omega\right\}, \mathcal{D}_{3} \equiv\left\{w \in L_{2}\left(S_{T}\right): w(x, t) \in W\right.$ п.в. на $\left.S_{T}\right\}$, где $U \subset \mathbb{R}^{1}, V \subset \mathbb{R}^{1}, W \subset \mathbb{R}^{1}$ - выпуклые компакты. С целью перехода к регуляризованному ПМП запишем ПМП в простейшей задаче оптимального управления с сильно выпуклым функционалом Лагранжа в качестве функционала качества (см., например, [5, лемма 7])

$$
L_{p}^{\delta}(\pi, \lambda) \equiv\|\pi\|^{2}+\left\langle\lambda, z^{\delta}[\pi](\cdot, T)-h^{\delta}-p\right\rangle \rightarrow \min , \quad \pi \in \mathcal{D} \subset \mathcal{H},
$$

при произвольном фиксированном $\lambda \in H$. Введем функции Гамильтона - Понтрягина:

$$
H_{u}(u, \eta) \equiv \eta u-u^{2}, \quad H_{v}(v, \eta) \equiv \eta v-v^{2}, \quad H_{w}(w, \eta) \equiv \eta w-w^{2} .
$$

Лемма 3. Тройка $\pi^{\delta}[\lambda] \equiv\left(u^{\delta}[\lambda], v^{\delta}[\lambda], w^{\delta}[\lambda]\right)$ удовлетворяет обычному ПМП в задаче (4.4): для $\pi \equiv(u, v, w)=\pi^{\delta}[\lambda]$ выполняются соотношения максимума

$$
\begin{gathered}
\max _{r \in U} H_{u}\left(x, t, r, \eta^{\delta}[\lambda](x, t)\right)=H_{u}\left(x, t, u(x, t), \eta^{\delta}[\lambda](x, t)\right) \quad \text { для n.в. }(x, t) \in Q_{T}, \\
\max _{r \in V} H_{v}\left(x, r, \eta^{\delta}[\lambda](x, 0)\right)=H_{v}\left(x, v(x), \eta^{\delta}[\lambda](x, 0)\right) \text { для n.в. } x \in \Omega, \\
\max _{r \in W} H_{w}\left(s, t, r, \eta^{\delta}[\lambda](s, t)\right)=H_{w}\left(s, t, w(s, t), \eta^{\delta}[\lambda](s, t)\right) \text { для n.в. }(s, t) \in S_{T},
\end{gathered}
$$

где $\eta^{\delta}[\lambda] \in V_{2}^{1,0}\left(Q_{T}\right)-$ решение сопряженной задачи

$$
\begin{gathered}
-\eta_{t}-\frac{\partial}{\partial x_{j}}\left(a_{i, j}^{\delta}(x, t) \eta_{x_{i}}\right)+a^{\delta}(x, t) \eta=0, \quad \eta(x, T)=\lambda(x), \quad x \in \Omega, \\
\frac{\partial \eta}{\partial \mathcal{N}}+\sigma^{\delta}(x, t) \eta=0, \quad(x, t) \in S_{T} .
\end{gathered}
$$

Обратно, в силу выпуклости задачи $\left(O C_{p}^{\delta}\right)$ любал тройка $\pi \in \mathcal{D}$, удовлетворлющая при некотором $\lambda \in \mathcal{H}$ соотношениям (4.5), (4.6), дает минимум в задаче (4.4).

Д о к а з а т е л ь с т в о более общего по сравнению с леммой 3 утверждения см. в $[27$, теорема 4.1].

Обозначим через $\pi_{m}^{\delta}[\lambda]$ элемент $\pi \in \mathcal{D}$, удовлетворяющий всем соотношениям ПМП (4.5) леммы 3. Очевидно, в условиях данной работы $\pi_{m}^{\delta}[\lambda]=\pi^{\delta}[\lambda]$. Благодаря лемме 3 регуляризованный итерационный ПЛ теоремы 6 может быть в этом случае ограниченного $\mathcal{D}$ переписан в форме регуляризованного ПМП в итерационной форме.

Теорема 8 [ПМП в итерационной регуляризованной форме]. Для существования минимизирующего приближенного решения в задаче $\left(O C_{p}^{0}\right)$ необходимо и достаточно, чтобы для 
последовательности двойственной переменной $\bar{\lambda}^{k}, k=0,1,2, \ldots$, порождаемой итерачионным прочессом

$$
\bar{\lambda}^{k+1}=\bar{\lambda}^{k}+\beta^{k}\left(A^{\delta^{k}} \pi_{m}^{\delta^{k}}\left[\bar{\lambda}^{k}\right]-h^{\delta^{k}}-p\right)-2 \beta^{k} \alpha^{k} \bar{\lambda}^{k}, \quad k=0,1,2, \ldots, \quad \bar{\lambda}^{0} \in H,
$$

с условиями согласования (3.3), выполнялись соотношения

$$
\pi_{m}^{\delta^{k}}\left[\bar{\lambda}^{k}\right] \in \mathcal{D}_{p}^{\delta^{k}, \epsilon^{k}}, \quad \epsilon^{k} \rightarrow 0, \quad\left\langle\bar{\lambda}^{k}, A^{\delta^{k}} \pi_{m}^{\delta^{k}}\left[\bar{\lambda}^{k}\right]-h^{\delta^{k}}-p\right\rangle \rightarrow 0, \quad k \rightarrow \infty
$$

При этом последовательность $\pi_{m}^{\delta^{k}}\left[\bar{\lambda}^{k}\right], k=1,2, \ldots$, представляет собою искомое минимизирующее приближенное решение в задаче $\left(O C_{p}^{0}\right)$ и вне зависимости от того, разрешима или нет двойственная $\kappa\left(O C_{p}^{0}\right)$ задача, $\pi_{m}^{\delta^{k}}\left[\bar{\lambda}^{k}\right] \rightarrow \pi_{p}^{0}, k \rightarrow \infty$ (см. лемму 2). Более того, выполняется предельное соотношение $V_{p}^{0}\left(\bar{\lambda}^{k}\right) \rightarrow \sup _{\lambda \in H} V_{p}^{0}(\lambda)=\left\|\pi_{p}^{0}\right\|^{2}, k \rightarrow \infty$.

Как и теорема 6, теорема 8 снабжается соответствующим регуляризирующим правилом останова итерационного процесса (4.7) в случае, когда исходные данные задачи $\left(O C_{p}^{0}\right)$ задаются с фиксированной конечной ошибкой, характеризуемой величиной $\delta>0$. Оно почти дословно совпадает с правилом останова теоремы 7 и может быть использовано для практического решения неустойчивых задач на основе устойчивого итерационного ПМП теоремы 8 , так как представляет собою устойчивый алгоритм построения минимизирующего приближенного решения в задаче $\left(O C_{p}^{0}\right)$. Пусть последовательности $\delta^{k}, \alpha^{k}, \beta^{k}, k=0,1,2, \ldots$, удовлетворяют условиям согласования (3.3). Зададим правило останова итерационного процесса (4.7)

$$
\bar{\lambda}^{k+1}=\bar{\lambda}^{k}+\beta^{k}\left(A^{\delta} \pi_{m}^{\delta}\left[\bar{\lambda}^{k}\right]-h^{\delta}-p\right)-2 \beta^{k} \alpha^{k} \bar{\lambda}^{k}, \quad k=0,1,2, \ldots, \quad \bar{\lambda}^{0} \in H,
$$

с фиксированной конечной ошибкой $\delta>0$ следующим образом: для каждого $\delta>0$ такого, что $\delta \leq \delta^{1}$, итерации продолжаются до такого наибольшего номера $k=k(\delta)$, для которого выполняются неравенства (3.6). Следствием теорем 5 и 7 является

Теорема 9. Справедливы предельные соотношения $\pi_{m}^{\delta}\left[\bar{\lambda}^{k(\delta)}\right] \rightarrow \pi_{p}^{0}, V_{p}^{0}\left(\bar{\lambda}^{k(\delta)}\right) \rightarrow \sup _{\lambda \in H} V_{p}^{0}(\lambda)$ при $\delta \rightarrow 0$, где $\bar{\lambda}^{k(\delta)}$ - результат $k(\delta)$ итераций итерационного прочесса $(4.8)$ с правилом останова (3.6).

\section{Заключение}

В статье обсуждены так называемые регуляризованные КУО для задач ВП, оптимального управления и обратных задач. Они сформулированы как теоремы существования минимизирующих последовательностей - минимизирующих приближенных решений в смысле Дж. Варги, выражаются в терминах регулярных обычных функций Лагранжа и Гамильтона - Понтрягина и представляют собою регуляризирующие алгоритмы для решения оптимизационных задач с одновременным конструктивным представлением сходящихся к решениям задач минимизирующих последовательностей. Устроенные структурно так же, как и классические ПМ и ПМП, регуляризованные ПЛ и ПМП:

1) преодолевают возможные свойства некорректности классических КУО;

2) приводят к классическим аналогам "в пределе";

3) являются теоретической базой для конструирования на своей основе численных алгоритмов для практического решения различных конкретных оптимизационных и обратных задач. 


\section{СПИСОК ЛИТЕРАТУРЫ}

1. Сумин М.И. Регуляризованная параметрическая теорема Куна-Таккера в гильбертовом пространстве // Журн. вычисл. математики и мат. физики. 2011. Т. 51, № 9. С. 1594-1615.

2. Сумин М.И. Устойчивое секвенциальное выпуклое программирование в гильбертовом пространстве и его приложение к решению неустойчивых задач // Журн. вычисл. математики и мат. физики. 2014. Т. 54, № 1. С. 25-49. doi: 10.7868/S0044466914010141.

3. Васильев Ф.П. Методы оптимизации: в 2-х кн. Москва: МЦНМО, 2011. 1056 с.

4. Алексеев В.М., Тихомиров В.М., Фомин С.В. Оптимальное управление. Москва: Наука, 1979. 432 c.

5. Сумин М.И. Регуляризованный градиентный двойственный метод решения обратной задачи финального наблюдения для параболического уравнения // Журн. вычисл. математики и мат. физики. 2004. Т. 44, № 11. С. 2001-2019.

6. Сумин М.И. Регуляризация в линейно выпуклой задаче математического программирования на основе теории двойственности // Журн. вычисл. математики и мат. физики. 2007. Т. 47, № 4. С. 602-625.

7. Сумин М.И. Параметрическая двойственная регуляризация для задачи оптимального управления с поточечными фазовыми ограничениями // Журн. вычисл. математики и мат. физики. 2009. T. 49, № 12. C. 2083-2102.

8. Варга Дж. Оптимальное управление дифференциальными и функциональными уравнениями. Москва: Наука, 1977. 624 с.

9. Sumin M.I. Regularization of Pontryagin maximum principle in optimal control of distributed systems // Ural Math. J. 2016. Vol. 2, iss. 2. P. 72-86. doi: https://doi.org/10.15826/umj.2016.2.008.

10. Sumin M.I. Regularized Lagrange principle and Pontryagin maximum principle in optimal control and inverse problems // IFAC PapersOnLine. 2018. Vol. 51, iss. 32. P. 871-876. doi: https://doi.org/10.1016/j.ifacol.2018.11.435.

11. Сумин М.И. Зачем нужна регуляризация принципа Лагранжа и принципа максимума Понтрягина и что она дает // Вестн. Тамбов. ун-та. Сер. Естественные и технические науки. 2018. Т. 23, вып. 124. С. 757-775. doi: 10.20310/1810-0198-2018-23-124-757-775 .

12. Сумин М.И. Некорректные задачи и методы их решения. Материалы к лекциям для студентов старших курсов. Нижний Новгород: Изд-во Нижегород. гос. ун-та, 2009. 289 с.

13. Сумин М.И. Субоптимальное управление системами с распределенными параметрами: минимизирующие последовательности, функция значений // Журн. вычисл. математики и мат. физики. 1997. Т. 37, № 1. С. 23-41.

14. Смирнов В.И. Курс высшей математики. Т. 5. Москва: Гос. изд-во физ.-мат. лит., 1959. 656 с.

15. Loewen P.D. Optimal control via nonsmooth analysis. CRM Proc. and Lecture Notes. Vol. 2. Providence, RI: Amer. Math. Soc., 1993. 158 p.

16. Крейн С.Г. Линейные уравнения в банаховом пространстве. Москва: Наука, 1971. 104 с.

17. Треногин В.А. Функциональный анализ. Москва: Наука, 1980. 496 с.

18. Ладыженская О.А., Солонников В.А., Уральцева Н.Н. Линейные и квазилинейные уравнения параболического типа. Москва: Наука, 1967. 736 с.

19. Плотников В.И. Энергетическое неравенство и свойство переопределенности системы собственных функций // Изв. АН СССР. Сер. математическая. 1968. Т. 32, № 4. С. 743-755.

20. Плотников В.И. Теоремы единственности, существования и априорные свойства обобщенных решений // Докл. АН СССР. 1965. Т. 165, № 1. С. 33-35.

21. Кутерин Ф.А., Сумин М.И. О регуляризованном принципе Лагранжа в итерационной форме и его применении для решения неустойчивых задач // Мат. моделирование. 2016. Т. 28 , № 11. C. $3-18$.

22. Кутерин Ф.А., Сумин М.И. Устойчивый итерационный принцип Лагранжа в выпуклом программировании как инструмент для решения неустойчивых задач // Журн. вычисл. математики и мат. физики. 2017. Т. 57, № 1. С. 55-68. doi: 10.7868/S0044466917010100 .

23. Калинин А.В., Сумин М.И., Тюхтина А.А. Об обратных задачах финального наблюдения для системы уравнений Максвелла в квазистационарном магнитном приближении и устойчивых секвенциальных принципах Лагранжа для их решения // Журн. вычисл. математики и мат. физики. 2017. Т. 57, № 2. С. 187-209. doi: 10.7868/S0044466917020089 .

24. Обен Ж.-П. Нелинейный анализ и его экономические приложения. Москва: Мир, 1988. 264 с. 
25. Кутерин Ф.А., Сумин М.И. Регуляризованный итерационный принцип максимума Понтрягина в оптимальном управлении I: оптимизация сосредоточенной системы // Вестн. Удмурт. ун-та. Математика. Механика. Компьютерные науки. 2016. Т. 26, вып. 4. С. 474-489. doi: 10.20537/vm160403.

26. Кутерин Ф.А., Сумин М.И. Регуляризованный итерационный принцип максимума Понтрягина в оптимальном управлении II: оптимизация распределенной системы // Вестн. Удмурт. ун-та. Математика. Механика. Компьютерные науки. 2017. Т. 27, вып. 1. С. 26-41. doi: 10.20537/vm170103.

27. Сумин М.И. Принцип максимума в теории субоптимального управления распределенными системами с операторными ограничениями в гильбертовом пространстве // Итоги науки и техники. Сер. Соврем. математика и ее приложения. Темат. обзор. ВИНИТИ. 1999. Т. 66. С. 193-235.

Поступила 14.12.2018

После доработки 14.02.2019

Сумин Михаил Иосифович

Принята к публикации 26.02.2019

д-р физ.-мат. наук, профессор

профессор

Нижегородский государственный университет им. Н. И. Лобачевского

г. Нижний Новгород

e-mail: m.sumin@mail.ru

\section{REFERENCES}

1. Sumin M.I. Regularized parametric Kuhn-Tucker theorem in a Hilbert space. Comput. Math. Math. Phys., 2011, vol. 51, no. 9, pp. 1489-1509. doi: 10.1134/S0965542511090156.

2. Sumin M.I. Stable sequential convex programming in a Hilbert space and its application for solving unstable problems. Comput. Math. Math. Phys., 2014, vol. 54, no. 1, pp. 22-44. doi: 10.1134/S0965542514010138.

3. Vasil'ev F.P. Metody optimizatsii [Optimization methods]. Moscow: MTsNMO Publ., 2011. Vol. 1: 620 p., ISBN: 978-5-94057-707-2 ; Vol. 2: 433 p., ISBN: 978-5-94057-708-9.

4. Alekseev V.M., Tikhomirov V.M., Fomin S.V. Optimal control. N Y: Plenum Press, 1987, 309 p. doi: 10.1007/978-1-4615-7551-1.

5. Sumin M.I. A regularized gradient dual method for the inverse problem of a final observation for a parabolic equation. Comput. Math. Math. Phys., 2004, vol. 44, no. 11, pp. 1903-1921.

6. Sumin M.I. Duality-based regularization in a linear convex mathematical programming problem. Comput. Math. Math. Phys., 2007, vol. 47, no. 4, pp. 579-600. doi: 10.1134/S0965542507040045.

7. Sumin M.I. Parametric dual regularization for an optimal control problem with pointwise state constraints. Comput. Math. Math. Phys., 2009, vol. 49, no. 12, pp. 1987-2005. doi: 10.1134/S096554250912001X.

8. Warga J. Optimal control of differential and functional equations. N Y: Acad. Press, 1972, 531 p. ISBN: 0127351507. Translated to Russian under the title Optimal'noe upravlenie differentsial'nymi $i$ funktsional'nymi uravneniyami. Moscow: Nauka Publ., 1977, 624 p.

9. Sumin M.I. Regularization of Pontryagin maximum principle in optimal control of distributed systems. Ural Math. J., 2016, vol. 2, no. 2, pp. 72-86. doi: 10.15826/umj.2016.2.008.

10. Sumin M.I. Regularized Lagrange principle and Pontryagin maximum principle in optimal control and inverse problems. IFAC PapersOnLine, 2018, vol. 51, no. 32, pp. 871-876. doi: 10.1016/j.ifacol.2018.11.435 .

11. Sumin M.I. Why regularization of Lagrange principle and Pontryagin maximum principle is needed and what it gives. Vestnik Tambov. Univ. Ser. Estestvennye i Tekhnicheskie Nauki, 2018, vol. 23, no. 124, pp. 757-775 (in Russian). doi: 10.20310/1810-0198-2018-23-124-757-775 .

12. Sumin M.I. Nekorrektnye zadachi i metody ikh resheniya. Materialy $k$ lektsiyam dlya studentov starshikh kursov: Uchebnoe posobie [Ill-posed problems and their solution methods. Materials for lectures for senior students: Textbook]. Nizhnii Novgorod: Nizhnii Novgorod State Univ. Publ., 2009, 289 p.

ISBN: 978-5-91326-160-1.

13. Sumin M.I. Suboptimal control of distributed-parameter systems: Minimizing sequences and the value function. Comput. Math. Math. Phys., 1997, vol. 37, no. 1, pp. 21-39. 
14. Smirnov V.I. A course of higher mathematics. Vol. V. Oxford: Pergamon, 1964, 638 p. ISBN: 9781483139371. Original Russian text published in Sminnov V.I. Kurs vysshei matematiki. Vol. 5. Moscow: Gos. Izd-vo Fiz.-Mat. Lit., 1959, 656 p.

15. Loewen P.D. Optimal control via nonsmooth analysis. CRM Proc. and Lecture Notes, vol. 2. Providence, RI: Amer. Math. Soc., 1993, 158 p. ISBN: 0-8218-6996-5.

16. Krein S.G. Linear equations in Banach spaces. Boston, Basel, Stuttgart: Birkhaüser, 1982,105 p. doi: 10.1007/978-1-4684-8068-9. Original Russian text published in Krein S.G. Lineinye uravneniya $v$ banakhovom prostranstve. Moscow: Nauka Publ., 1971, 104 p.

17. Trenogin V.A. Funktsional'nyi analiz [Functional analysis]. Moscow: Nauka Publ., 1980, 496 p. ISBN: 5020148911 .

18. Ladyzhenskaya O.A., Solonnikov V.A., Ural'tseva N.N. Linear and quasilinear equations of parabolic type. Providence, R.I.: AMS, 1968, 648 p. ISBN: 978-0-8218-1573-1. Original Russian text published in Ladyzhenskaya O.A., Solonnikov V.A., Ural'tseva N.N. Lineinye $i$ kvazilineinye uravneniya parabolicheskogo tipa. Moscow: Nauka Publ., 1967, 736 p.

19. Plotnikov V.I. An energy inequality and the overdeterminacy property of a system of eigenfunctions. Math. USSR-Izv., 1968, vol. 2, no. 4, pp. 695-707. doi: 10.1070/IM1968v002n04ABEH000656.

20. Plotnikov V.I. Uniqueness and existence theorems and apriori properties of generalized solutions. Sov. Math., Dokl., 1965, vol. 6, pp. 1405-1407.

21. Kuterin F.A., Sumin M.I. On the regularized Lagrange principle in iterative form and its application for solving unstable problems. Mathematical Models and Computer Simulations, 2017, vol. 9, no. 3, pp. 328-338. doi: 10.1134/S2070048217030085.

22. Kuterin F.A., Sumin M.I. Stable iterative Lagrange principle in the convex programming as a tool for solving unstable problems. Comput. Math. Math. Phys., 2017, vol. 57, no. 1, pp. 71-82. doi: 10.1134/S0965542517010092 .

23. Kalinin A.V., Sumin M.I., Tyukhtina A.A. Inverse final observation problems for Maxwell's equations in the quasi-stationary magnetic approximation and stable sequential Lagrange principles for their solving. Comput. Math. Math. Phys., 2017, vol. 57, no. 2, pp. 189-210. doi: 10.1134/S0965542517020075.

24. Aubin J.P. L'analyse non linéaire et ses motivations économiques. Paris: Masson, 1984, 214 p. Translated to Russian under the title Nelineinyi analiz i ego ekonomicheskie prilozheniya. Moscow: Mir Publ., 1988, $264 \mathrm{p}$.

25. Kuterin F.A., Sumin M.I. The regularized iterative Pontryagin maximum principle in optimal control. I. Optimization of a lumped system. Vestnik Udmurt. Univ. Matematika. Mekhanika. Komp'yuternye Nauki, 2016, vol. 26, no. 4, pp. 474-489 (in Russian). doi: 10.20537/vm160403.

26. Kuterin F.A., Sumin M.I. The regularized iterative Pontryagin maximum principle in optimal control. II. Optimization of a distributed system. Vestnik Udmurt. Univ. Matematika. Mekhanika. Komp'yuternye Nauki, 2017, vol. 27, no. 1, pp. 26-41 (in Russian). doi: 10.20537/vm170103.

27. Sumin M.I. Maximum principle in suboptimal control theory of distributed-parameter systems with operator constraints in a Hilbert space. J. Math. Sci., 2001, vol. 104, no. 2, pp. 1060-1086. doi: 10.1023/A:1009535725511.

Received December 14, 2018

Revised February 14, 2019

Accepted February 26, 2019

Funding Agency: This work was supported by the Russian Foundation for Basic Research (project no. 19-07-00782).

Mikhail Iosifovich Sumin, Dr. Phys.-Math. Sci., Prof., Nizhnii Novgorod State University, Nizhnii Novgorod, 603950 Russia, e-mail: m.sumin@mail.ru . 